\title{
EFEITO DA RETIRADA DA INFLORESCÊNCIA MASCULINA NA PRECOCIDADE DA COLHEITA E PRODUÇÃO DA BANANEIRA-'PRATA-ANÃ' SOB IRRIGAÇÃO NA REGIÃO NORTE DE MINAS GERAIS ${ }^{1}$
}

\author{
ROSILENE FERREIRA SOUTO², MARIA GERALDA V. RODRIGUES ${ }^{3}$, JOÃO L. P. MENEGUCCI ${ }^{4}$
}

\begin{abstract}
RESUMO: O trabalho foi desenvolvido na região Norte de Minas Gerais, em uma área pertencente a EPAMIG - CTNM, localizada no Perímetro Irrigado do Jaíba. O delineamento experimental utilizado foi inteiramente casualizado, com dois tratamentos e 22 repetições, e cada parcela constituída por três plantas. Objetivou-se avaliar o efeito da retirada da inflorescência masculina na precocidade de colheita e produção da bananeira-'Prata-Anã', avaliando-se os três primeiros ciclos. Houve redução no período entre a retirada da inflorescência e a colheita, quando se eliminou a inflorescência masculina, sendo que, no primeiro ciclo, promoveu aumento no peso total de frutos, peso médio de frutos, peso médio de pencas, diâmetro e peso do fruto central e espessura de casca do fruto central da segunda penca, peso da penca 2, peso da penúltima penca, peso do engaço e peso e comprimento da ráquis. Não promoveu aumento de peso total do cacho, no comprimento do fruto central da segunda penca, peso da penca 1 e peso da última penca. No segundo ciclo, apesar de tendência de aumento, não houve diferença estatística para os parâmetros de produção. Já no terceiro ciclo, houve aumento no peso do cacho, além de aumento nos parâmetros de produção, concordando com os resultados obtidos na primeira colheita.
\end{abstract}

Termos para indexação: Bananeira, Musa sp., manejo do cacho, poda de coração, Prata-Anã, Inflorescência masculina.

\section{INFLUENCE OF MALE FLOWERAGE PRUNING ON THE IRRIGATED BANANA BUNCH CROP IN THE NORTH OF MINAS GERAIS STATE, BRASIL}

\begin{abstract}
This work was carried out in the EPAMIG/CTNM's Experimental Farm, which is located in the irrigated area of Jaiba county in the north of Minas Gerais state, Brazil. The experimental design was completely randomized with two treatments and 22 replicants and each plot had three plants. The aim of this work was to evaluate the influence of male flowerage pruning on the bunch crop of irrigated banana 'Prata Ana' in the three first crops. The male flowerage pruning made the harvest time shorter and in the first crop the characteristics as total fruit weight; mean fruit weight, second fruit of the second hand girth and weight; second hand weight; hand before the last hand weight; peduncle's weight and length was bigger than in the non-flowerage pruned banana tree plants. There was no response of pruning for the total bunch weight, first hand and last hand weight, and length of the central fruit of the second hand. In the second crop there was no response for the crop characteristics. In the third crop there was improvement of crop's characteristics as in the first crop.
\end{abstract}

Index terms: Musa sp., bunch trait, male flowerage prune, 'Prata-Anã'.

\section{INTRODUÇÃO}

A região Norte de Minas Gerais, com clima semi-árido semelhante ao do Nordeste brasileiro, tornou-se alvo de investimentos públicos em irrigação a partir da década de 70 , dentro dos vários programas nacionais instituídos pelo governo federal, visando ao desenvolvimento da região, atingida seriamente pelo problema da seca.

A bananicultura foi introduzida na região após os primeiros testes realizados pela EPAMIG e, posteriormente, pelos produtores do Perímetro Irrigado do Gorutuba, situados em Porteirinha-MG. Os primeiros plantios ocorreram já no início da década de 80 , com a variedade Nanicão, que sofreu intenso ataque de nematóides, comprometendo a sua viabilidade na região. Gradativamente, esta cultivar foi substituída pela 'Prata-
Anã', devido a sua grande aceitação, melhor remuneração e qualidade de frutos (Souto et al., 1997).

As técnicas de manejo do cacho na pré-colheita, visando à melhoria da qualidade e precocidade da produção, são bastante difundidas nos países que trabalham tradicionalmente com a bananeira. Esses países investem na qualidade dos frutos como resposta a um mercado extremamente exigente, existindo, portanto, uma grande demanda de pesquisas nesta área, resultando em técnicas como a retirada da inflorescência masculina. Estas pesquisas, porém, são feitas basicamente com bananas do subgrupo Cavendish, por serem estas as mais comuns na bananicultura de exportação, conhecidas mundialmente. No Brasil, esses trabalhos ainda não são muito comuns, por se considerar que o mercado não é tão exigente na qualidade dos frutos. No entanto, existem alguns trabalhos realizados com as

1 Trabalho $\mathrm{n}^{\mathbf{0}}$ 169/2000. Recebido: 03/08/2000. Aceito para publicação: 11/07/2001.

2 Eng. Agrônoma, MS., Pesquisadora EPAMIG/CTTP Uberaba-MG.

3 Eng. Agrônoma, MS., Pesquisadora EPAMIG/CTNM Janaúba-MG.

4 Eng. Agrônomo, Dr., Pesquisador EMBRAPA/Mandioca e Fruticultura/EPAMIG Uberaba-MG 
cultivares Nanica, Nanicão, Prata comum e Terra, sob condições de sequeiro. Há, portanto, escassez de trabalhos com a 'PrataAnã' irrigada, cultivar muito difundida e plantada hoje em várias regiões do Brasil.

Pesquisadores trabalhando com diferentes cultivares de bananeira concluíram que a retirada da inflorescência masculina acelera o processo de desenvolvimento dos frutos (Simmonds, 1961; Nóbrega, 1967; Pereira et al., 1981; Vitti e Ruggiero, 1984; Manica e Gomes, 1984; Moreira, 1987; Lichtemberg et al., 1991; Durigan e Ruggiero, 1995). No entanto, em trabalhos realizados com a 'Prata' comum e com a bananeirada-'Terra', não foi observado este efeito (Gregory, 1955; Souza et al.,1971, e Pereira et al., 1981).

Em relação à produção, a retirada da inflorescência masculina aumentou o peso do cacho (Durigan e Ruggiero, 1995; Moreira, 1987; Manica e Gomes, 1984; Boncato, 1970; Champion, 1963; Simmonds, 1961; Trupim, 1959; Wills, 1951) e tamanho do cacho (Manica e Gomes, 1984 e Borges et al., 1997). Além destes parâmetros, Lichtemberg et al. (1991) observaram aumento no tamanho dos frutos. Esses resultados diferem dos encontrados por Pereira et al. (1981), Souza et al. (1971), Sampaio e Simão (1970) e Gregory (1955) que não observaram aumento de peso do cacho.

Segundo Simmonds (1961), a retirada da inflorescência melhora a qualidade dos frutos. Esta prática visa também melhoria da forma dos frutos (Manica e Gomes, 1984), com aumento de comprimento dos mesmos (Moreira, 1987; Durigan e Ruggiero, 1995). Monge (1984), citado por Soto (1992), observou melhora no índice de curvatura das frutas, tornando-as mais retas. Já Souza et al. (1971) não observaram aumento no comprimento e diâmetro dos frutos quando trabalharam com a 'Prata' comum.

Segundo Manica e Gomes (1984), Vitti e Ruggiero (1984), Nóbrega (1967), Trupim (1959) e Wills (1951), a retirada da inflorescência reduz o peso total do cacho, reduzindo assim o tombamento da planta.

A retirada da inflorescência masculina é recomendada também para o controle de doenças como o Moko (Soto,1992, e Manica e Gomes, 1984) e pragas como o tripes (Borges et al., 1997; Souto et al., 1997; Durigan e Ruggiero, 1995; Manica e Gomes, 1984; Vitti e Ruggiero, 1984; e Lozano et al.,1970) e a traça da bananeira (Moreira, 1987).

Simmonds (1961) salienta que, enquanto esta prática de remoção da inflorescência masculina é comum em Queensland, ela é proibida por lei em New South Wales, visto que é na inflorescência masculina que a doença conhecida como "bunchtop" pode ser prontamente reconhecida.

Esta prática facilita a ocorrência de podridões no eixo floral (Manica e Gomes, 1984). Champion (1963) observou que, apesar de ser uma prática corrente nas Canárias, nas estações úmidas ocorrem podridões no interior da ráquis, comprometendo a conservação do cacho após a colheita. Pereira et al.(1981), Souza et al. (1971) e Sampaio e Simão (1970), trabalhando com as cultivares Terra, Prata comum e Nanicão, respectivamente, não observaram esse problema.

Além dos efeitos diretos sobre o cacho, Vitti e Ruggiero (1984) relatam que a inflorescência eliminada, a ráquis e o engaço podem ser aproveitados como fonte de nutrientes, particularmente de potássio, ou pela disponibilidade e mineralização rápida dos restos vegetais, reduzindo assim a quantidade de adubos minerais a ser utilizada. Além destes benefícios, Simmonds (1961) relata que a parte masculina da inflorescência da bananeira é removida para servir de alimento aos animais.

Este trabalho foi desenvolvido com o objetivo de verificar o efeito da retirada da inflorescência masculina da 'PrataAnã' sobre as características dos frutos e precocidade de produção.

\section{MATERIAL E MÉTODOS}

O trabalho foi conduzido na Fazenda Experimental de Mocambinho (FEMO) pertencente a EPAMIG (CTNM), localizada no Perímetro Irrigado do Jaíba, localizado no extremo Norte do Estado de Minas Gerais entre $43^{\circ} 29^{\prime}$ e $44^{\circ} 6^{\prime}$ de longitude, $14^{\circ} 33^{\prime}$ e $15^{\circ} 28^{\prime}$ de latitude Sul com altitude de aproximadamente $500 \mathrm{~m}$. A pluviosidade média anual é de aproximadamente de $871 \mathrm{~mm}$, a temperatura média anual é de $24^{\circ} \mathrm{C}$, a insolação é de 2763 horas anuais e a umidade relativa média é de 70,6\%. O solo foi classificado como Latossololo Vermelho-Amarelo Distrófico, com $22 \%$ de argila, $68 \%$ de areia e $10 \%$ de silte (classe textural franco-argilo-arenosa) e cultivado pela primeira vez. Foi feita amostragem do solo antes do preparo da área. As devidas correções foram feitas com aração seguida de calagem, gradagem e preparo dos sulcos. Adicionaram-se 3,2 litros de esterco de galinha por metro linear de sulco, formaram-se as covas com adição de fosfato e micronutrientes.

As mudas obtidas por cultura de tecidos foram repicadas para sacos de polietileno, onde permaneceram por 30 dias e então levadas ao campo. O plantio foi feito no dia 20-0796 , em covas de $40 \times 40 \times 40 \mathrm{~cm}$ abertas sobre sulcos de $30 \mathrm{~cm}$ de profundidade, no espaçamento $3,0 \times 2,7 \mathrm{~m}$.

A irrigação foi realizada com aspersão convencional nos primeiros 45 dias, sendo então substituída por microaspersão. Como o solo possui baixa capacidade de retenção de água, a irrigação foi feita diariamente, calculando-se a lâmina de água com base na evaporação do Tanque Classe $\mathrm{Ae}$ Kc da cultura. Utilizou-se Kc recomendado por Doorembos e Kassam (1994), porém mantendo o valor 1, a partir do oitavo mês. Todos os dados climáticos necessários ao cálculo da irrigação foram obtidos na estação meteorológica do INMET localizada na FEMO.

O delineamento experimental utilizado foi inteiramente casualizado, com 2 tratamentos, 22 repetições e 3 plantas por parcela. Os tratamentos foram representados por poda da inflorescência masculina (sem inflorescência) e sem poda da inflorescência masculina (com inflorescência). A retirada da inflorescência foi feita quebrando a inflorescência quando à distância entre esta e a última penca atingia 15 a $20 \mathrm{~cm}$.

A colheita foi feita quando os cachos apresentavam os frutos no estádio "de vez", quando ocorreram as mudanças da tonalidade dos frutos de verde-escuro para verde-claro.

Foi feito controle de broca e sigatoka amarela através de monitoramento. Foram avaliados os seguintes parâmetros, nos três primeiros ciclos de produção: dias entre o ponto de retirada da inflorescência e a colheita, peso do cacho $(\mathrm{kg})$, peso total de frutos $(\mathrm{kg})$, peso médio de frutos $(\mathrm{g})$, peso médio de pencas $(\mathrm{kg})$, comprimento do fruto central da segunda penca 
TABELA 1. Efeito da retirada da inflorescência masculina nos três ciclos de produção da banana-Prata-Anã, cultivada sob irrigação, no Norte de Minas Gerais, 1998.

\begin{tabular}{|c|c|c|c|c|c|c|c|c|c|c|c|c|}
\hline & \multicolumn{4}{|c|}{ Primeiro Ciclo } & \multicolumn{4}{|c|}{ Segundo Ciclo } & \multicolumn{4}{|c|}{ Terceiro Ciclo } \\
\hline Parâmetros avaliados & $\begin{array}{l}\text { Com } \\
\text { inflorescência }\end{array}$ & & $\begin{array}{l}\text { Sem } \\
\text { inflorescência }\end{array}$ & & $\begin{array}{l}\text { Com } \\
\text { Inflorescência }\end{array}$ & & $\begin{array}{l}\text { Sem } \\
\text { inflorescência }\end{array}$ & & $\begin{array}{l}\text { Com } \\
\text { Inflorescência }\end{array}$ & & $\begin{array}{l}\text { Sem } \\
\text { inflorescência }\end{array}$ & \\
\hline Dias da retirada da inflorescência à colheita & 130,00 & $\mathrm{a}$ & 119,00 & $\mathrm{~b}$ & 122,00 & $\mathrm{a}$ & 109,00 & $\mathrm{~b}$ & 119,00 & a & 117,00 & $\mathrm{a}$ \\
\hline Peso do cacho $(\mathrm{kg})$ & 10,39 & $\mathrm{a}$ & 10,97 & $\mathrm{a}$ & 14,91 & $\mathrm{a}$ & 15,83 & $\mathrm{a}$ & 20,21 & $\mathrm{~b}$ & 23,78 & $\mathrm{a}$ \\
\hline Peso total de frutos $(\mathrm{kg})$ & 10,22 & $\mathrm{~b}$ & 11,93 & $\mathrm{a}$ & 12,84 & a & 13,54 & a & 18,00 & $\mathrm{~b}$ & 21,33 & $\mathrm{a}$ \\
\hline Peso médio de frutos $(\mathrm{g})$ & 83,46 & $\mathrm{~b}$ & 94,59 & $\mathrm{a}$ & 108,18 & a & 113,36 & $\mathrm{a}$ & 107,10 & $\mathrm{~b}$ & 125,00 & $\mathrm{a}$ \\
\hline Peso médio de pencas ( $\mathrm{kg}$ ) & 1,21 & $\mathrm{~b}$ & 1,35 & a & 1,46 & a & 1,54 & a & 1,69 & $\mathrm{~b}$ & 1,96 & a \\
\hline Comprimento fruto central da $2^{\mathrm{a}}$ penca $(\mathrm{cm})$ & 14,39 & $\mathrm{a}$ & 13,30 & $\mathrm{a}$ & 13,16 & $\mathrm{a}$ & 13,67 & $\mathrm{a}$ & 13,11 & $\mathrm{~b}$ & 14,51 & $\mathrm{a}$ \\
\hline Diâmetro fruto central segunda penca $(\mathrm{cm})$ & 3,15 & $\mathrm{~b}$ & 3,27 & $\mathrm{a}$ & 3,39 & $\mathrm{a}$ & 3,42 & $\mathrm{a}$ & 3,24 & $\mathrm{~b}$ & 3,38 & a \\
\hline Peso do fruto central da segunda penca $(\mathrm{g})$ & 102,64 & $\mathrm{~b}$ & 115,48 & $\mathrm{a}$ & 115,31 & a & 122,73 & $\mathrm{a}$ & 118,33 & $\mathrm{~b}$ & 137,96 & a \\
\hline Espessura casca fruto central $2^{\mathrm{a}}$ penca $(\mathrm{mm})$ & 0,30 & $\mathrm{~b}$ & 0,33 & a & 0,30 & a & 0,31 & a & 0,29 & a & 0,30 & $\mathrm{a}$ \\
\hline Peso penca $1(\mathrm{~kg})$ & 1,85 & $\mathrm{a}$ & 1,78 & $\mathrm{a}$ & 1,93 & a & 1,86 & $\mathrm{a}$ & 2,4 & a & 2,6 & $\mathrm{a}$ \\
\hline Peso penca $2(\mathrm{~kg})$ & 1,54 & $\mathrm{~b}$ & 1,76 & $\mathrm{a}$ & 1,67 & $\mathrm{a}$ & 1,82 & $\mathrm{a}$ & 2,1 & $\mathrm{a}$ & 2,4 & $\mathrm{a}$ \\
\hline Peso penúltima penca $(\mathrm{kg})$ & 1,12 & $\mathrm{~b}$ & 1,25 & $\mathrm{a}$ & 1,36 & a & 1,38 & $\mathrm{a}$ & 1,40 & $\mathrm{~b}$ & 1,62 & a \\
\hline Peso última penca $(\mathrm{kg})$ & 0,84 & a & 0,87 & a & 0,98 & a & 0,90 & a & 0,93 & a & 1,07 & $\mathrm{a}$ \\
\hline Peso médio dos frutos da última penca & 70,45 & $\mathrm{~b}$ & 83,81 & $\mathrm{a}$ & 86,54 & a & 89,10 & $\mathrm{a}$ & 0,08 & $\mathrm{~b}$ & 0,09 & $\mathrm{a}$ \\
\hline Peso engaço $(\mathrm{kg})$ & 2,54 & $\mathrm{a}$ & 2,60 & $\mathrm{a}$ & 2,07 & $\mathrm{a}$ & 2,29 & $\mathrm{a}$ & 2,31 & $\mathrm{a}$ & 2,44 & $\mathrm{a}$ \\
\hline
\end{tabular}

As médias seguidas pela mesma letra, na mesma linha, não diferem ente si, pelo teste de Tukey, a 5\% de probabilidade.

$(\mathrm{cm})$, diâmetro do fruto central da segunda penca $(\mathrm{cm})$, diâmetro do fruto central da segunda penca $(\mathrm{cm})$, peso do fruto central da segunda penca $(\mathrm{g})$, espessura de casca do fruto central da segunda penca $(\mathrm{mm})$, peso da penca $1(\mathrm{~kg})$, peso penca $2(\mathrm{~kg})$, peso da penúltima penca $(\mathrm{kg})$, peso da última penca $(\mathrm{kg})$, peso do engaço $(\mathrm{kg})$, peso da ráquis $(\mathrm{kg})$ e comprimento da ráquis $(\mathrm{cm})$. Foi feita análise estatística dos dados e análise das médias pelo teste de Tukey.

\section{RESULTADOS E DISCUSSÃO}

Avaliados os dados, foram obtidos, nos três primeiros ciclos, os resultados apresentados na Tabela 1. Os dados do primeiro ciclo demonstraram resposta positiva à retirada da inflorescência, para os parâmetros dias entre o ponto da retirada da inflorescência e a colheita, peso total de frutos, peso médio de frutos, peso médio de pencas, diâmetro do fruto central da segunda penca, peso do fruto central da segunda penca, espessura de casca do fruto central da segunda penca, peso da penca 2, peso da penúltima penca, peso médio dos frutos da última penca, peso do engaço, confirmando as afirmações feitas por Borges et al. (1997), Lichtemberg et al. (1991), Moreira (1987) e Manica e Gomes (1984).

Portanto, a retirada da inflorescência masculina aumentou o rendimento do cacho, produziu frutos com maior diâmetro e antecipou a colheita em 11 dias. Esses resultados provavelmente ocorreram em função da eliminação do dreno de fotoassimilados representado pela inflorescência masculina, que, para a 'Prata-Anã' é exageradamente grande e que, neste caso, teve peso médio de $1,56 \mathrm{~kg}$.

Não houve diferença significativa para o peso da última penca; no entanto, houve uma grande diferença entre o número de frutos da última penca dos cachos avaliados, variação esta que alcançou até $50 \%$ em torno do número médio (12 frutos). Esta variação indica a presença de frutos falsos, que podem ter influenciado o resultado, uma vez que houve resposta ao tratamento para peso médio dos frutos da última penca da plantamãe e neta, e tendência de aumento na planta-filha.

Não houve diferença também para comprimento do fruto central da penca 2 e peso da penca 1 . Como a retirada da inflorescência é feita cerca de 20 dias após a antese, o comprimento dos frutos das pencas 1 e 2 já está praticamente definido. Estes resultados concordam com as observações feitas por Soto (1992), quando afirma que o comprimento final dos frutos é alcançado num período de 30 a 35 dias após a floração, nas variedades do subgrupo Cavendish. Segundo Medina et al. (1985), amadurece uma penca por dia, sendo que a diferença de idade das pencas está relacionada com o número de pencas do cacho.

Como era esperado, o segundo ciclo apresentou produtividade superior ao primeiro, com peso total de frutos 19 $\%$ maior. Porém, só respondeu positivamente ao tratamento o parâmetro precocidade de produção, sendo que a retirada da inflorescência masculina reduziu o ciclo em 13 dias. Apesar de não haver diferença estatística, houve tendência de aumento para os outros parâmetros, exceto para peso da penca 1 e peso da última penca, confirmando os resultados do primeiro ciclo. As plantas do segundo ciclo floresceram em junho/ julho, e as baixas temperaturas que ocorreram no período de crescimento destes cachos, cuja média mínima de julho foi de $13,6^{\circ} \mathrm{C}$, podem ter reduzido o crescimento delas e ter sido responsável pela resposta não significativa aos tratamentos. Já no terceiro ciclo, houve aumento no peso do cacho, além de aumento nos parâmetros de produção, concordando com os resultados obtidos na primeira colheita.

\section{CONCLUSÕES}

1 - Com a retirada da inflorescência masculina, houve aumento de rendimento do cacho representado por peso total e médio de frutos, e diâmetro médio dos frutos no primeiro e terceiro ciclos. Na colheita do terceiro ciclo, houve aumento no peso do cacho, além de aumento no peso total e médio de frutos, peso médio de pencas, comprimento, diâmetro e peso do fruto central da segunda penca, peso médio dos frutos da última penca, peso da penúltima penca, concordando com os resultados obtidos na primeira colheita. Apesar da tendência de aumento, o segundo ciclo não apresentou resposta aos tratamentos, para os parâmetros de produção.

2 - Houve redução no período entre floração e colheita, 
nos dois primeiros ciclos de produção, quando a inflorescência masculina foi retirada. Esta tendência também foi observada no terceiro ciclo, mas não houve diferença estatística.

\section{REFERÊNCIAS BIBLIOGRÁFICAS}

BONCATO, A .A. Effects of reducing the number of hands in a bunch of Lacatan banana. Phillips. Journal of Plant Industry., Phillippine, v. 32, p. 243-51, 1967.

BORGES, A L.; ALVES, E. J.; SILVA, S . de O; SOUZA, L. da S.; MATOS, A P. de; FANCELLI, M.; OLIVEIRA, A M. G.; CORDEIRO, Z. J. M.; SILEIRA, J. R. S.; COSTA, D. da C.; MEDINA, V. M.; OLIVEIRA, S. L. de; SOUZA, J. das S. OLIVEIRA, R. P. de; CARDOSO, C. E. L.; MATSUURA, F. C. A U.; ALMEIDA, C. O de. O Cultivo da banana. Cruz das Almas: EMBRAPACNPMF, 1997. 109 p. (Circular Técnica, 27).

CHAMPION, J. Le bananier. Paris. Maisonneuve \& Larose, 1963. $263 \mathrm{p}$.

DURIGAN, J. F.; RUGGIERO, C. Bananas de Qualidade. Jaboticabal: FUNEP, 1995. 37 p.

GREGORY, E. J. Investigations. Jamaica: Dep. Agric., 1955. v. 54, 1320 p. 1955.

LICHTEMBERG, L. A.; ZAFFARI, G. R.e HINZ, R. H. Experimentos preliminares sobre poda da inflorescência masculina e poda de pencas em banana-'Nanicão'. Revista Brasileira de Fruticultura, Cruz das Almas, v.13, n.2, p.9-18, 1991.

LOZANO, T. J. C.; THURSTON, H. D.; GALVEZ, R. G. E. Control del "moko"del plátano y banano causado por la bactéria Pseudomonas solanacearum. Agricultura Tropical'Bogotá, 25 p.315-24. 1969. In: Horticultural Abstracts, England 40(2):579, Abstr. 4835. 1970.

MANICA,I.; GOMES, J. A; Outras Práticas Culturais Importantes. In: SIMPÓSIO BRASILEIRO SOBRE BANANICULTURA, 1., 1984, Jaboticabal, Anais... p.196-213.
MOREIRA, R. S. Banana: Teoria e Prática de Cultivo. Campinas, Fundação Cargill, 1987. 335 p.

NÓBREGA N. R. As culturas de bananeiras na Austrália. O Estado de São Paulo, São Paulo, 4 outubro 1967. Suplemento Agrícola, p. 15.

PEREIRA, L. V.; ALVES, E. J.; LUCCHINI, F. Influência da Época de Corte da Inflorescência Masculina da Bananeira'Terra'sobre a uniformidade dos frutos. Cruz das Almas: EMBRAPA-CNPMF, 1981. 16p. (Boletim de pesquisa, 3).

SIMMONDS, N. W. Bananas 2. ed. London. Longmanss Green, $1961.512 \mathrm{p}$.

SAMPAIO, V. R.; SIMÃO, S. Banana - Remoção da Inflorescência Masculina em Cachos Novos. Revista de Agricultura, Piracicaba, v.45, n.2/3, p. 93-95. 1970.

SOTO BALLESTERO, M. Bananos: cultivo y comercialización. 2.ed. San José, Costa Rica: Litografia e Imprenta Lil, 1992. 674 p.

SOUTO, R. F; RODRIGUES, M. G. V.; ALVARENGA, C. D.; SILVA, J. T. A.da; MAENO, P.; GONZAGA, V. Sistema de Produção para a Cultura da Banana-Prata-Anã, no Norte de Minas. Belo Horizonte: EPAMIG, 1997. 32 p. (Boletim Técnico, 48).

SOUZA, M. M.de; ANDERSEN, O; MANICA, I. Influência do Corte da Parte Terminal da Ráquis do Cacho da Bananeira cv. 'Prata'(Musa sp.)Sobre a Produção Total, Número de Pencas por Cacho. Comprimento e Diâmetro do Fruto. Ceres, Viçosa, v. 18, p. 98, p.315-325, 1971.

TRUPIN, F. Coupe du bourgeon male ser I'inflorescence du bananier Gross Michel. (Paris). Fruits, v. 14, n. 9: p. 389-90, 1959.

VITTI, G. C.; RUGGIERO, C. Aproveitamento do engaço, coração e ráquis, como fonte de nutrientes. In: SIMPÓSIO BRASILEIRO DE BANANICULTURA,1., 1984, Jaboticabal, SP. Anais... Jaboticabal: UNESP, 1984. p. 392-9.

WILLS, I. N. Banana growing in Queesland Agricultural Journal, Brisbane, v. 72, p.147-58, p.223-36. 